Introduction An estimated 180,000 people in the UK are chronically infected with hepatitis B (HBV). NICE guidelines published in 2013 advocate changes in the management of this patient group. Recommendation toward use of peginterferon alfa-2a (PEG-IFN) as first line therapy, a reduced threshold for treatment initiation, and the monitoring of chronically infected individuals with annual elastography hold particular resource implications.

University Hospitals Bristol (UHB) serves a largely urban population of approximately 350,000, encompassing large immigrant communities, including a Somali population of approximately 10,000 . UHB provides an elastography service to an additional 3 district hospitals.

Through analysis of our existing HBV patient population the resource implications of adopting 2013 NICE guidelines, in contrast to 2012 EASL guidelines, are assessed.

Methods Local HBV databases were scrutinised to identify all HBV patients attending over a one year period from november 2012. Patients defaulting follow-up during this period were excluded. A cost of treatment analysis was undertaken by reassessing each patient's treatment eligibility according to 2013 NICE guidance. Additional monitoring and clinic follow up costs were also calculated.

Results 154 patients were identified, 93 of whom were receiving antiviral nucleos ( $\mathrm{t}$ )ide analogue treatment (24 initiated). 2 had initiated PEG-IFN. 61 were under monitoring only.

All 24 patients starting therapy met NICE criteria for PEG-IFN, the excess cost of which was $£ 137,003$ over a 1 year period. The estimated additional number of treatment clinic visits for this group is 202 (assuming an extra 8.4 visits/patient initiated). This analysis does not include increased demand for liason psychiatry and virology services. There would be 109 extra elastography appointments required for this cohort alone, amounting to a further 28 clinics a year. 3 district general hospitals currently refer patients to UHB for elastography, and this analysis does not account for the inevitable increase in such referrals.

Conclusion Implementation of 2013 NICE guidelines for management of chronic HBV represent a significant challenge to NHS resources. In addition to increased drug expenditure, markedly increased clinic capacity and specialist nurse provision will be required in our centre to facilitate increased used of PEG-IFN and to provide sufficient transient elastography for our region. Additional demands on virology and liason psychiatry services are also likely. Practical suggestions for improving efficiency and capacity, such as 'one stop' fibroscan and follow up clinics, are discussed.

Disclosure of Interest None Declared.

\section{PTH-079 TENOFOVIR AND ENTECAVIR IN THE TREATMENT OF CHRONIC HEPATITIS B: EFFECTIVENESS AND SAFETY IN A SINGLE CENTRE EXPERIENCE}

DK Hanumantharaya*, R Khan, M Wilkinson, T Wong, G Tritto. Gastroenterology, Guy's and St Thomas Hospital, London, UK

\subsection{6/gutjnl-2014-307263.525}

Introduction Tenofovir and Entecavir are direct acting antivirals used in the treatment of chronic hep B infection. We describe our hospital experience of the effectiveness of these antiviral drugs in terms of HbeAg seroconversion, development of drug resistence and incidence of hepatocellular carcinoma during treatment.
Methods All patients treated with Tenofovir or Entecavir between 2010 and 2013 at Guy's and St Thomas hospital trust were identified from hospital pharmacy database. Baseline HBV DNA levels, ALT and albumin levels were recorded on three monthly intervals for upto 48 months.

Results A total of 132 patients were identified. 84 patients were on Tenofovir (17 pregnant women). Among them, 38\% ( $\mathrm{n}=$ 32) were HbeAg positive, and $26 \%(n=22)$ were cirrhotic. The remaining 48 patients were on Entecavir. Of these, 27\% ( $\mathrm{n}=$ 13) were HbeAg positive and $31 \%(\mathrm{n}=15)$ were cirrhotic when treatment was started. The mean baseline HBV DNA levels for HbeAg positive patients were $7.0 \log 10 \mathrm{IU}(\mathrm{P}=0.0170)$ and $5.4 \log 10 \mathrm{IU}(\mathrm{P}=0.0153)$ for Tenofovir and Entecavir respectively. The mean baseline HBV DNA levels in cirrhotic patients were $4.6 \log 10 \mathrm{IU}$ and $4.7 \log 10 \mathrm{IU}$ for Tenefovir and Entecavir patients respectively.

Sub group analysis showed that HbeAg negative patients achieved early undetectable viral loads (mean 2.1 months on tenefovir, mean 2.8 months on entecavir). HbeAg positive patients achived undetectable viral loads relatively later (mean 11 months on Tenefovir,mean 9 months on Entecavir).

HbeAg seroconversion was achieved in 19\% $(n=6)$ of patients on Tenofovir and 15\% $(n=2)$ of patients on Entecavir. Treatment with both antivirals was associated with improvement in synthetic function in cirrhotic patients. Drug resistance occurred in one patient on Entecavir and none of the patients on Tenefovir. Four patients ( 2 patients on Tenofovir and 2 on Entecavir) developed hepatocellular carcinoma during treatment, all of whom were cirrhotic prior to treatment.

Among the 17 pregnant patients treated with Tenofovir, 10 patients were HbeAg positive. The mean pre-treatment baseline HBV DNA levels were $6.8 \log 10 \mathrm{IU}$. HbeAg seroconversion was noted in 2 patients.

Conclusion Treatment of chronic hepatitis B infection with both Tenofovir and Entecavir were well tolerated. Undetectable viral loads were achieved in both groups within 12 months of starting treatment. HbeAg seroconversion was noted in 19\% $(\mathrm{n}=6)$ patients treated with Tenofovir and $15 \%(\mathrm{n}=2)$ patients treated with Entecavir. Overall there was no significant difference in the mean time to undetectable viral loads in patients treated with Tenofovir (7.5 months) and Entecavir (6.1 months).

\section{REFERENCE}

Marecellin P, Heathcote EJ, Buti M, et al. NICE-Entecavir for the treatment of chronic hepatitis $B$.

Disclosure of Interest None Declared.

\section{PTH-080 RISK PROFILE FOR NON-ALCOHOLIC FATTY LIVER DISEASE (NAFLD) IN A PAEDIATRIC SPECIALIST CARE SETTING}

${ }^{1}$ FWD Tai*, ${ }^{1} Y$ Derwa, ${ }^{2} \mathrm{~S}$ Naik, ${ }^{1} \mathrm{~W}$ Alazawi. ${ }^{1}$ Department of Gastroenterology, Blizzard Institute, Queen Mary University of London, London, UK; ${ }^{2}$ Department of Paediatric Gastroenterology, Barts Health NHS Trust, London, UK

\subsection{6/gutjnl-2014-307263.526}

Introduction Non-alcoholic fatty liver disease (NAFLD) is a major cause of liver dysfunction in adults. Much less is known about this disease in children, although obesity and metabolic dysfunction are believed to be risk factors. In England the prevalence rates of obesity in children in Reception classes (age 4) and Year 6 (age 11) are $10.8 \%$ and $22.4 \%{ }^{[1]}$ respectively in 2012/ 13. In inner city populations such as those served by The Royal 